\title{
Wildlife translocation: the conservation implications of pathogen exposure and genetic heterozygosity
}

\author{
Walter M Boyce ${ }^{1 *}$, Mara E Weisenberger ${ }^{2}$, M Cecilia T Penedo $^{3}$, Christine K Johnson ${ }^{1}$
}

\begin{abstract}
Background: A key challenge for conservation biologists is to determine the most appropriate demographic and genetic management strategies for wildlife populations threatened by disease. We explored this topic by examining whether genetic background and previous pathogen exposure influenced survival of translocated animals when captive-bred and free-ranging bighorn sheep (Ovis canadensis) were used to re-establish a population that had been extirpated in the San Andres Mountains in New Mexico, USA.

Results: Although the free-ranging source population had significantly higher multi-locus heterozygosity at 30 microsatellite loci than the captive bred animals, neither source population nor genetic background significantly influenced survival or cause of death. The presence of antibodies to a respiratory virus known to cause pneumonia was associated with increased survival, but there was no correlation between genetic heterozygosity and the presence of antibodies to this virus.

Conclusions: Although genetic theory predicts otherwise, increased heterozygosity was not associated with increased fitness (survival) among translocated animals. While heterosis or genetic rescue effects may occur in F1 and later generations as the two source populations interbreed, we conclude that previous pathogen exposure was a more important marker than genetic heterozygosity for predicting survival of translocated animals. Every wildlife translocation is an experiment, and whenever possible, translocations should be designed and evaluated to test hypotheses that will further improve our understanding of how pathogen exposure and genetic variability influence fitness.
\end{abstract}

\section{Background}

Innate and adaptive immune responses evolved in vertebrates as a first and secondary line of defense, respectively, against a diverse and changing array of pathogenic organisms. The effectiveness of these immunologic responses, and hence the fitness of individuals, populations, and species, is driven by pathogen exposure history and the immunogenetic repertoire of major histocompatibility complex (MHC) genes and non-MHC genes $[1,2]$. Novel, highly virulent pathogens can overwhelm host immune responses not primed to their exposure, and such pathogens can be a strong selective force, reducing the distribution and abundance of a

\footnotetext{
* Correspondence: wmboyce@ucdavis.edu

'Wildlife Health Center, University of California, One Shields Avenue, Davis, California 95616, USA

Full list of author information is available at the end of the article
}

species over short timeframes (1-2 generations) through effects on survival and reproductive success [2]. Over multiple generations, a history of ongoing pathogen exposure theoretically should select for more resistant immunogenotypes that limit fitness impacts by responding effectively upon initial exposure (innate immunity) or re-exposure (adaptive immunity).

Bighorn sheep (Ovis canadensis) are a useful model for examining this interplay between disease, demography, and genetics. They are a polygynous, highly philopatric species found in small, fragmented populations in the mountainous regions of western North America [3]. They are highly susceptible to infectious disease, and outbreaks of disease regularly cause high morbidity and mortality $[3,4]$. The history of population die-offs dates back to European settlement of the western United States $>200$ yrs ago [3], indicating that novel pathogens
C Biomed Central

() 2011 Boyce et al; licensee BioMed Central Ltd. This is an Open Access article distributed under the terms of the Creative Commons Attribution License (http://creativecommons.org/licenses/by/2.0), which permits unrestricted use, distribution, and reproduction in any medium, provided the original work is properly cited. 
were likely introduced by contact with domestic sheep (Ovis aries). Pneumonia epizootics appear to be driven by density-dependence, serving to constrain population size [5], and presumably selecting for the most fit genotypes. However, small populations of bighorn sheep also are prone to inbreeding and genetic drift, making it difficult to understand the relative importance of pathogen-mediated selection, drift, and inbreeding on genetic variability and fitness.

We approached this problem by testing whether genetic background and previous pathogen exposure influenced survival when animals from two different founder populations were simultaneously translocated into the San Andres Mountains (SAM) in New Mexico, USA. The SAM once supported the largest population of native bighorn sheep in the state. However, by the late 1990's, a combination of disease, mountain lion (Puma concolor) predation, and drought had reduced this population to the point of extinction, and translocation from captive and/or free-ranging herds was necessary to reestablish a self-sustaining population in the SAM. The two founder populations chosen for reintroduction were a genetically diverse free-ranging herd in the Kofa National Wildlife Refuge (KNWR), Arizona, and a less diverse captive herd in the Red Rock Wildlife Area (RRWA) that was originally derived from the native SAM population. In November 2002, 51 bighorn sheep were translocated into the SAM from the KNWR $(\mathrm{n}=20)$ and the RRWA $(\mathrm{n}=31)$, and 30 more bighorn were translocated from KNWR in November 2005. We examined genetic variation at 33 microsatellite loci to compare genetic variability, and we conducted population health analyses at the time of capture to assess infectious disease exposure. Radiocollared sheep released into the SAM were then monitored through February 2007 to determine survivorship and cause specific mortality.

\section{Methods}

\section{Study animals}

The SAM mountain range represents the largest amount of high quality bighorn sheep habitat in New Mexico, and the native SAM bighorn sheep population exceeded 200 animals prior to a psoroptic scabies epizootic that began in the late 1970s. The population remained around 30 animals through the early 1990's and then declined to a single ewe that was temporarily brought into captivity in 1999 for less than two weeks. Nine animals sampled just prior to extirpation of the population had low mean heterozygosity at MHC (0.075) and microsatellite (0.359) loci [6]. Immediately prior to the reintroduction effort that began in November 2002, the SAM population consisted of 4 rams that had been translocated from the RRWA as part of a sentinel disease study, and the single native ewe that was released along with the sentinel rams [7]. A total of 81 bighorn sheep were captured, sampled, and translocated to the SAM in 2002 and 2005 from RRWA and KNWR. In 2007, nine offspring from these animals were captured and sampled for genetics and disease surveillance in the SAM. The survival analysis presented in this paper focuses only on animals translocated to the SAM, and data from these 9 offspring born in the SAM are presented for descriptive purposes only.

The RRWA is a 500-hectare enclosure for bighorn sheep located in central New Mexico. The RRWA population was initiated in 1972 with founders from the SAM, and has served as the source for $>260$ bighorn sheep translocated within New Mexico since 1979 [8]. This population has been managed as a closed herd (no immigrants), and has low genetic diversity (0.36) relative to other free-ranging populations in the desert southwest (0.44-0.63) [9]. Genetic and disease samples for this study were collected when bighorn sheep were captured for translocation to the SAM in $2002(n=31)$. The size of the RRWA population was $<100$ animals at the time of capture and translocation.

The KNWR is located in southwestern Arizona and contains a free-ranging population of bighorn sheep that has significantly higher heterozygosity $(0.60)$ than the RRWA captive population [9]. Like the RRWA population, the KNWR herd has been the source for transplants of hundreds of animals within Arizona and to other western states. Genetic and disease samples for this study were collected at capture from animals translocated to the SAM in $2002(\mathrm{n}=21)$ and $2005(\mathrm{n}=30)$. The population at KNWR has averaged about 700-800 animals since 1981 , but dropped to $<400$ animals by 2006. Since $1979,>560$ bighorn sheep have been translocated from KNWR.

\section{Genetic analysis}

Genomic DNA was extracted from blood and genetic variability was assessed by examining 33 microsatellite loci (OarFCB128, MAF209, OarFCB304, MAF33, MAF48, MAF65, OarFCB11, MAF36, OarFCB266, ETH152 (= D5S2), DRB3 (MHC-linked loci), BMC1009, CELJP23, BM203, OARCP026, TGLA94, FCB193, IRBP, CELB9, BM6506, CELJP15, BM4107, CSRD247, HSC, INRA023, INRA063, INRA105, MAF214, McM527, OarAE129, OarCP49, OarFCB20, SPS113). Many of these loci have been examined in previous publications $[6,9,10]$, and we have used them to examine heterozygosity in different populations of bighorn sheep across their range in North America (unpublished). Briefly, using fluorescently labeled microsatellite primers, microsatellites present in genomic DNA were amplified by PCR (polymerase chain reaction). PCR products were 
separated by capillary electrophoresis using an Applied Biosystems 3730 DNA Analyzer (Applied Biosystems, Foster City, CA), with a fluorescent-labeled base pair size standard in each lane. Image analysis and fragment size determination were carried out using STRand software [11]. Deviations from Hardy-Weinberg equilibrium (HWE) and allele frequencies were examined with GenePop [12].

Multi-locus heterozygosity (MLH) values were calculated for each animal as the preferred metric of genetic variability. Chapman et al. [13] showed that different genetic metrics (MLH, SH, IR) are highly correlated and non-independent, and they advocated the use of the simplest metric, MLH, in future studies of heterozygotefitness correlations (HFC). For our multivariate analyses, MLH values were categorized into two categories using a cutoff $>0.5$ to indicate a generally low or high level of genetic diversity.

\section{Assessment of Health and Disease Exposure Status}

Antibodies are a marker of previous exposure and priming of the adaptive immune response to specific pathogens. Sera from blood collected at the time of capture were tested for the presence of antibodies to three viruses known to cause respiratory disease and pneumonia in bighorn sheep: bovine respiratory syncytial virus (BRSV), bluetongue virus (BTV), and parainfluenza-3 virus (PI3). Assays were performed at the California Animal Health and Food Safety laboratory in Davis, California, and results were classified as positive or negative. Results for BTV were reported as positive or negative; titers $\geq 1: 20$ were considered positive for BRSV, and titers $>1: 16$ were considered positive for PI3.

\section{Post-release Monitoring}

A VHF radiocollar (Telonics Inc., Lotek Wireless Inc., Advanced Telemetry Systems, Inc., and Telemetry Solutions) with mortality sensor was placed on animals to be translocated at the time of capture to facilitate monitoring after their release in the SAM. Animals were transported to the San Andres National Wildlife Refuge in the SAM and released within $48 \mathrm{hrs}$ of capture. Resight of individual animals by radio-signal was conducted by state and federal wildlife biologists through February 2007 to detect mortalities and determine specific causes of death. Mortalities were investigated immediately and a field investigation and necropsy typically performed within 72 hrs of death. Mortalities were classified as lion predation based on criteria of Hayes et al. [14], or as pneumonia based on gross lesions present in the lungs. All mortalities that could not be classified as predation or pneumonia based on field examination were categorized as unknown, but "other" than predation and pneumonia. The number of days each animal survived was calculated as the difference between the date of release and the date of death, or the date sheep were last observed. For individuals lost to follow up, the end date was the date individuals were last observed, and for sheep that were confirmed alive at the end of the study period, this date was February 1, 2007.

\section{Statistical Analysis}

We used independent univariate and multivariate approaches to explore correlation within our dataset, and to identify factors most influencing pathogen exposure, number of days until death (or end of study period if survived) and death due to suspected pneumonia. Independent variables evaluated for their impact on these three outcomes included sex, age-class $(<3 \mathrm{yr}$, 3 to $<6 \mathrm{yr}, \geq 6 \mathrm{yr}$ ), source population (RRWA vs KNWR), year released (2002, 2005), exposure status to each pathogen (BRSV, BTV, PI3) at time of release, and genetic variability (MLH). Significant associations with outcomes variables, confounding, and effect modification were evaluated by stratified univariate analyses using exact categorical tests and the student's t test. The relationship between MLH (as a continuous variable) and age and sex class were evaluated by the two-way ANOVA in order to adjust for source population. Independent categorical variables were also examined for their relationship to loss to follow-up by the two-sided Fisher exact test to determine whether variables were related to censorship, and therefore could not be included in the survival analyses.

Binary logistic regression was used to evaluate whether independent variables were related to death due to pneumonia, which was the most common cause of death detected during the study period. Only sheep that died of suspected pneumonia or were confirmed alive at the end of the study were included in this analysis $(\mathrm{n}=50)$. Sheep that died from predation or unknown causes were excluded from this analysis. Variables were selected by backward stepwise elimination (likelihood ratio test $\mathrm{P}<0.1$ ) and confidence intervals for the logistic model evaluating risk factors for pneumonia were calculated using conditional exact inference due to low sample size. Hosmer-Lemeshow goodness of fit test was used to estimate overall fit of the final logistic model.

For the survival analyses, median survival time was calculated for all risk factors of interest measured at time of translocation. The Kaplan-Meier product-limit estimator was used to estimate the survivor function, and the log-rank test [15] was used to test the equality of survivor function for each independent variable with groups considered significantly different if $\mathrm{P}<0.1$. Variables significantly associated with survival in these univariate analyses were evaluated for their relationship to failure rate (days until death) using the semi-parametric 
Cox proportional hazards regression. Sex was significantly associated with censorship so this variable was excluded from survival analyses. Variables were selected for the Cox proportional hazards model by manual backward elimination using a selection criterion of $\mathrm{P} \leq$ 0.1 for terms to stay in the model, and all categories of any significant variable were retained in the model. The Breslow approximation method was used to handle tied failure times [16].The proportional hazards assumption based on Schoenfeld residuals was evaluated to determine if the relative risk for each variable of interest was the same in time for the duration of the study. All statistical analyses were performed using STATA SE 11.1 software (STATACorp, 4905 Lakeway Drive, College Station, Texas 77845 USA).

\section{Results}

\section{Characteristics of Source Populations}

Bighorn sheep translocated from RRWA were predominantly male $(18 / 20)$, while $72 \%(34 / 47)$ of the sheep translocated from KNWR were female (Fisher exact $\mathrm{P}<$ 0.001). Age class was not significantly associated with source population (Fisher exact test $\mathrm{P}=0.068$ ), but twice as many sheep in the 3-6 yr old range were captured in KNWR (23/36) compared to RRWA (11/31). All sheep relocated to SAM in 2005 were from KNWR, but these newly introduced sheep were demographically similar and did not differ with regard to pathogen exposure or genetic diversity from sheep introduced from KNWR in 2002.

Serologic evidence of previous pathogen exposure varied substantially by source population for all pathogens, except PI3 (Table 1). Sheep from KNWR were more likely to be exposed to BRSV (14/36) compared to sheep

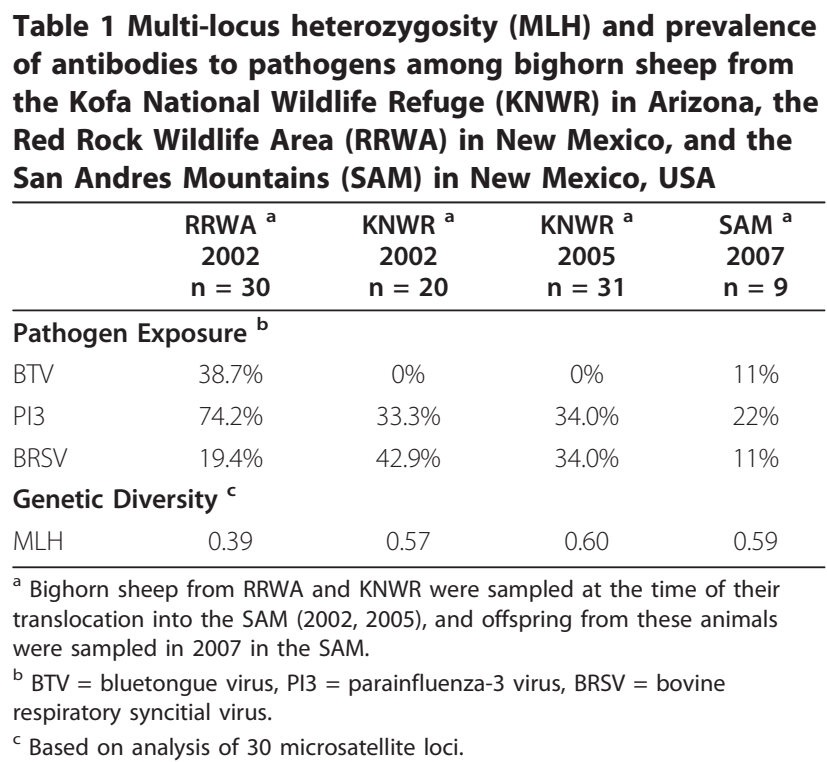

from RRWA (4/31, Fisher exact $\mathrm{P}=0.026)$. None of the sheep captured in KNWR had evidence of exposure to BTV, while 12/31 sheep from RRWA were seropositive to BTV (Fisher exact $\mathrm{P}<0.001$ ). Results from the univariate analysis stratified by source population indicated that age was a significant confounder related to BTV exposure for sheep from RRWA. In fact, older sheep ( $\geq 6$ years) captured at RRWA were 12 times more likely to be exposed to BTV than younger age classes (two sided Fisher exact test, $\mathrm{P}=0.012$ ). Significant associations with age class were not detected for the other pathogens after adjusting for the effect of source population.

Three of the 33 microsatellite loci tested were monomorphic and were excluded from further analyses (BM4107, INRA063, TGLA94). Complete genotypes were determined for all animals at each of the remaining 30 loci with the exception of a single animal at a single locus. Allele frequencies did not significantly differ from HWE expectations across loci, and null alleles were not detected. The genetic diversity parameter, MLH (Table 1), was significantly higher in sheep captured in KNWR (mean $=0.579,95 \%$ CI $0.555-0.603$ ) compared to sheep captured in RRWA (mean $=0.394,95 \% \mathrm{CI}=0.340$ 0.449). Mean MLH did not differ by sex or age class once comparisons were adjusted by source population.

\section{Specific Causes of Mortality}

Cause of death could be determined by field post-mortem examinations for 25 sheep. Pneumonia was suspected as a cause of mortality in 17 sheep, and mountain lion predation was identified as the cause of death in 8 sheep. Specific cause of death could not be determined for 9 sheep, but predation and pneumonia were excluded as their cause of death. Deaths due to lion predation occurred in 2002, 2003, and 2006, while deaths due to pneumonia occurred every year (2002-2007). Most deaths due to pneumonia $(65 \% ; 11 / 17)$ occurred in the fall from September thru November, and seven of these occurred in a cluster in the fall of 2006. Pneumonia impacted all age classes with $27 \%$ (4/15) of 1 -3 year old sheep, $35 \%$ $(9 / 26)$ of 3-6 year old sheep and $44 \%(4 / 9)$ of $>6$ year old sheep dying of pneumonia. Mean MLH was not significantly different among the 33 sheep that survived (mean $=0.50,95 \% \mathrm{CI} 0.45-0.55)$ compared to the 17 sheep that died of pneumonia (mean $=0.44,95 \%$ CI 0.37 - 0.51), but this analyses lacked power due to low sample size (two-sided t test $\mathrm{P}=0.161$, power $=0.31$ ). Similarly, risk factors were not significantly associated with death due to pneumonia in the multivariate analyses using exact conditional logistic regression.

\section{Risk Factors Associated with Survival}

Year of release was not associated with overall survival in univariate or multivariate analyses and survival time 
did not differ significantly by source population. However, age class at time of release was marginally associated with survivorship ( $\log$ rank test $P=0.079)$ in the univariate analyses. More than half of the sheep released at 1 to $<3$ years old survived the entire study period (1,509 days), while median survival time was 1,234 days for sheep 3 years to $<6$ years old and only 475 days for sheep released when 6 years or older. Genetic diversity and pathogen exposure were not related to survival time, even after stratifying by source population.

Risk factors found to be significantly associated with time to death in the Cox proportional hazards multivariate framework were the oldest age class and previous exposure to BRSV (Table 2). Based on the hazards ratios of the Cox proportional hazards model, we found that sheep with serological evidence of previous exposure to BRSV had approximately one-third the risk of death compared to sheep that had not been exposed to BRSV prior to release. Age was a significant confounding factor in the survival analyses, and as expected, older age class was associated with increasing risk and a shorter time to death. Sheep released at ages $\geq 6$ years had 3.4 times the hazard rate of sheep released at 1 to 3 years of age. Source population, MLH as a continuous and binary variable, year of release, and previous exposure to other pathogens were not associated with survival once we accounted for the influence of age and exposure to BRSV on time to death. Associations with sex could not be evaluated in the modeling procedure because males were more likely to be lost to follow up. Evaluation of the proportional hazards assumption for the final model based on a test of Schoenfeld residuals indicated that the relative risk for each variable of interest was the same in time for the duration of the study $(\mathrm{P}=0.322)$.

\section{Discussion}

A key challenge for conservation biologists is to determine the most appropriate demographic and genetic management strategies for wildlife populations threatened by disease [17-19].

\begin{tabular}{|c|c|c|c|c|c|}
\hline Factor & $\begin{array}{l}\text { Hazard } \\
\text { Ratio }\end{array}$ & $\begin{array}{l}\text { Std. } \\
\text { Err. }\end{array}$ & $z$ & $\mathrm{P}>|\mathrm{z}|$ & $\begin{array}{l}95 \% \text { Conf. } \\
\text { Interval }\end{array}$ \\
\hline $\begin{array}{l}\text { Age } 3 \text { to } \\
<6 \mathrm{yr}^{\mathrm{a}}\end{array}$ & 1.47 & 0.664 & 0.85 & 0.397 & $0.60-3.56$ \\
\hline Age $\geq 6 \mathrm{yr}^{\mathrm{a}}$ & 3.37 & 1.75 & 2.34 & 0.019 & $1.22-9.32$ \\
\hline $\begin{array}{l}\text { BRSV } \\
\text { antibodies b }\end{array}$ & 0.37 & 0.18 & -2.03 & 0.043 & $0.14-0.97$ \\
\hline
\end{tabular}

a Youngest age class (1 to $<3$ years old) designated as reference category.

${ }^{\mathrm{b}}$ BRSV = bovine respiratory syncitial virus.
Bighorn sheep in North America provide a useful model for examining this issue because they are highly susceptible to infectious diseases, and they are frequently translocated to re-establish or augment populations. In this study, we took advantage of a management effort - the restoration of bighorn sheep to the SAM to test whether genetic background and previous pathogen exposure influenced the survival of translocated bighorn sheep.

Variability and local adaptation are considered to be key genetic factors influencing the persistence of small populations of bighorn sheep $[10,20,21]$, and this strongly influenced the choice of source animals for the SAM translocation. Since the RRWA herd was founded with animals from the SAM, their translocation presumably maximized the retention of locally adapted gene complexes. However, the RRWA herd had low genetic variability (mean $M L H=0.39$; Table 1 ) because it had been managed for $>20$ years as an inbred population. In contrast, the KNWR population was much more diverse (mean MLH $=0.57-0.60$; Table 1 ), ensuring that the newly established SAM population would be more variable than one established only with animals from the RRWA captive population.

While the genetic management goal of achieving increased heterozygosity in the new SAM population was accomplished, it was not associated with enhanced survivorship of the individual sheep that were translocated. Bighorn sheep from the KNWR had significantly higher genetic diversity than those from the RRWA, but neither source population nor genetic background (MLH) influenced their survival in the SAM. Our failure to detect a significant relationship between genetic diversity and adult survival is consistent with the Chapman et al. [13] meta-analysis of HFC studies. In their comprehensive analysis, Chapman et al. [13] concluded that the effects of such correlations are very weak, and that other proposed measures of genetic variation $(\mathrm{SH}$, IR) are no more powerful than MLH for detecting relationships. We acknowledge that a larger sample size and more marker loci would have increased the power of our analysis, but any such effect is apparently very weak. Our failure to detect a fitness effect with neutral markers (microsatellites) is perhaps not surprising given that Gutierrez et al. [22] failed to find a strong association between MHC variation and disease resistance in bighorn sheep.

Previous examination of genetic variability among bighorn sheep suggests that microsatellite diversity has been influenced primarily by neutral factors, and MHC diversity by balancing selection $[6,9,22,23]$. These studies have shown that genetic distances between populations are roughly proportional to geographic distance, and that most genetic variability is apportioned within, 
rather than between, populations. These results are best explained by isolation by distance (reduced gene flow), and provide little genetic evidence for local adaptation. Our results are consistent with this view - the RRWA animals derived from the original SAM population did not have increased survivorship. Conversely, inbreeding within the RRWA population could have reduced fitness by increasing the accumulation and expression of deleterious recessive alleles $[24,25]$. We found no evidence of such an effect among translocated adult sheep (survival was not associated with source population). Although we did not detect fitness differences among translocated animals, heterosis or genetic rescue effects may occur in F1 and later generations as the two source populations interbreed $[25,26]$. While we were able to sample nine offspring born in the SAM for genetic and pathogen analyses (Table 1), we were unable to monitor their relative fitness and they were not included in any of our survival analyses.

Pneumonia was the most frequent cause of death, accounting for $50 \%(17 / 34)$ of the documented mortalities. This mirrors what has been reported for many other populations, illustrating the ongoing importance of pneumonia as a cause of morbidity and mortality across the range of bighorn sheep $[4,20,27,28]$. Several bacteria (Pasteurella and Manheimia spp.) and viruses (BRSV, PI3) have been implicated as primary or secondary pathogens in enzootic and epizootic pneumonia, and we associate these organisms with pneumonic disease because they can be detected in sick and dead animals. However, we know far less about causal relationships. The multifactorial etiology of pneumonia, coupled with the complex interplay of host, pathogen, and environment, has made it very difficult to identify a single, specific cause of pneumonia.

Instead of focusing on identifying pathogens after death, we looked at how previous pathogen exposure influenced the future survival of healthy bighorn sheep. The presence of antibodies was used as a marker of pathogen exposure, indicating that viral transmission and infection had occurred at some point in the past. Animals translocated into the SAM showed evidence of previous exposure to BTV (RRWA only) as well as BRSV and PI3 (both RRWA and KNWR; Table 1). All animals were healthy at the time of translocation, yet previous BRSV exposure was associated with increased survival regardless of age class. This suggests that antibodies to this virus provided some level of protection against pneumonia after animals were released into the SAM.

Age class at the time of release was significantly related to survival, and younger animals lived longer than those $>6$ yrs of age (Table 2). This intuitive result illustrates the demographic advantage of selecting young animals for reintroductions. Singer et al. [20] found that translocation success was directly related to the number of animals - larger translocations resulted in populations that were more likely to persist over time. However, pneumonia epizootics appear to be driven by densitydependence, and thus disease may constrain population growth and size [5]. Lethal removal of mountain lions was conducted in the SAM throughout our study period [8], likely influencing the relative proportion of mortality caused by predation versus pneumonia. In the absence of controlled studies, we have no way of inferring whether lion control increased overall survivorship. Regardless, there were no significant relationships between mean MLH and death due to lion predation or pneumonia.

\section{Conclusions}

We conclude that increased heterozygosity did not increase survival following translocation, nor did it reduce individual susceptibility to dying specifically from pneumonia. Instead, we found that previous pathogen exposure was more important than genetic heterozygosity as a marker for predicting survival of translocated animals. In their review of $>600$ published HFC effects, Chapman et al. [13] reported a publication bias with non-significant effects being under-reported in the literature. We acknowledge that confounding factors, such as age, and small sample sizes likely limited our ability to detect significant relationships between genetic variability, disease and fitness. These limitations often plague investigators who conduct observational field studies in natural settings. We encourage others to test the theoretical advantages of increased genetic variability using a variety of genetic markers in long term field studies that also evaluate ecological factors, such as disease, that are likely to influence survival. Every wildlife translocation is an experiment, but many translocations lack an experimental design. Whenever possible, translocations should be designed and evaluated to test hypotheses that will improve our understanding of how pathogen exposure and genetic variability influence fitness.

\section{Acknowledgements}

This project was supported by the U.S. Fish and Wildlife Service, New Mexico Department of Game and Fish (NMDGF), White Sands Missile Range, and the National Fish and Wildlife Foundation. We appreciate the support and cooperation of Elise Goldstein, Eric Rominger, and Darrel Weybright from NMDGF; Grace Lee from the University of California, Davis, as well as personnel from Arizona Department of Game and Fish, Arizona Desert Bighorn Sheep Society, and the New Mexico Chapter of Foundation for North American Wild Sheep. This study was performed in compliance with state and federal regulations.

The findings and conclusions in this article are those of the author(s) and do not necessarily represent the views of the U.S. Fish and Wildlife Service.

\section{Author details}

${ }^{1}$ Wildlife Health Center, University of California, One Shields Avenue, Davis, California 95616, USA. ${ }^{2}$ U.S. Fish and Wildlife Service, San Andres National 
Wildlife Refuge, 5686 Santa Gertrudis Drive, Las Cruces, New Mexico 88012, USA. ${ }^{3}$ Veterinary Genetics Laboratory, University of California, One Shields Avenue, Davis, California 95616, USA.

\section{Authors' contributions}

WMB and MEW designed and implemented the study. MCTP and CKJ conducted genetic and statistical analyses, respectively. All authors have approved the final manuscript.

Received: 27 April 2010 Accepted: 1 February 2011

Published: 1 February 2011

\section{References}

1. Acevedo-Whitehouse $K$, Cunningham AA: Is MHC enough for understanding wildlife immunogenetics? Trends Ecology Evolution 2006, 21:433-438.

2. Smith KF, Acevedo-Whitehouse K, Pederson AB: The role of infectious disease in biological conservation. Animal Conservation 2009, 12:1-12.

3. Buechner HK: The bighorn sheep of the United States: its past, present, and future. Wildlife Monographs 1960, 4:1-174.

4. Cassirer EF, Sinclair ARE: Dynamics of pneumonia in a bighorn sheep metapopulation. Journal Wildlife Management 2007, 71:1080-1088.

5. Monello RJ, Murray DL, Cassirer EF: Ecological correlates of pneumonia epizootics in bighorn sheep herds. Canadian Journal Zoology 2001 79:1423-1432.

6. Boyce WM, Hedrick PW, Muggli-Cockett NE, Kalinowski S, Penedo MCT, Ramey RR: Genetic variation of major histocompatibility complex and microsatellite loci: a comparison in bighorn sheep. Genetics 1996 145:421-433.

7. Boyce WM, Weisenberger $M$ : The rise and fall of psoroptic scabies in bighorn sheep in the San Andres Mountains, New Mexico. Journal Wildlife Diseases 2005, 41:525-531.

8. Rominger EM, Whitlaw HA, Weybright DL, Dunn WC, Ballard WB: The influence of mountain lion predation on bighorn sheep translocations. Journal Wildlife Management 2004, 68:993-999.

9. Gutiérrez-Espeleta GA, Kalinowski ST, Boyce WM, Hedrick PW: Genetic variation and population structure in desert bighorn sheep: implications for conservation. Conservation Genetics 2000, 1:3-15.

10. Whittaker DG, Ostermann SD, Boyce WM: Genetic variability of reintroduced California bighorn sheep in Oregon. Journal Wildlife Management 2004, 68:850-859.

11. Hughes SS: STRand Nucleic Acids Analysis Software. University of California, Davis, CA, USA; 2000.

12. Raymond M, Rousset F: GENEPOP 1.02: population genetics software for exact tests and ecumenicism. Journal Heredity 1995, 86:248-249.

13. Chapman JR, Nakagawa S, Coltman DW, Slate J, Sheldon BC: A quantitative review of heterozygote-fitness correlations in animal populations. Molecular Ecology 2009, 18:2746-2765.

14. Hayes $C L$, Rubin ES, Jorgensen MC, Botta RA, Boyce WM: Mountain lion predation of bighorn sheep in the Peninsular Ranges, California. Journal Wildlife Management 2000, 64:954-959.

15. Mantel $\mathrm{N}$, Haenszel W: Statistical aspects of the analysis of data from retrospective studies of disease. Journal National Cancer Institute 1959, 22:719-748.

16. Breslow N: Covariance analysis of censored survival data. Biometrics 1974, 30:89-99.

17. Caro TM, Laurenson MK: Ecological and genetic factors in conservation: a cautionary tale. Science 1994, 263:485-486.

18. Kinnison MT, Hendry AP, Stockwell CA: Contemporary evolution meets conservation biology II: impediments to integration and application. Ecological Research 2007, 22:947-954.

19. Pedersen $A B$, Jones $K E$, Nunn $C L$, Altizer S: Infectious disease and extinction risk in wild mammals. Conservation Biology 2007, 21:1269-1279.

20. Singer FJ, Papouchis C, Symonds K: Translocations as a tool for restoring bighorn sheep. Restoration Ecology 2000, 8:6-13.

21. United States Fish and Wildlife Service: Recovery plan for bighorn sheep in the Peninsular Ranges, California. U.S. Fish and Wildlife Service, Portland, Oregon; 2000.

22. Gutierrez-Espeleta GA, Hedrick PW, Kalinowski S, Garrigan D, Boyce WM: Is the decline of desert bighorn sheep from infectious disease the result of low MHC variation? Heredity 2001, 86:439-450.
23. Forbes SH, Hogg JT, Buchanan FC, Crawford AM, Allendorf FW: Microsatellite evolution in congeneric mammals: domestic and bighorn sheep. Molecular Biology Evolution 1995, 12:1106-1113.

24. Hedrick PW, Gutierrez-Espeleta GA, Lee RN: Founder effect in an island population of bighorn sheep. Molecular Ecology 2001, 10:851-857.

25. Tallman DA, Luikart G, Waples RS: The alluring simplicity and complex reality of genetic rescue. Trends Ecology Evolution 2004, 19:489-496.

26. Hogg JT, Forbes SH, Steele BM, Luikart G: Genetic rescue of an insular population of large mammals. Proceedings Royal Society B 2006, 273:1491-1499.

27. Gross JE, Singer FJ, Moses ME: Effect of disease, dispersal and area on bighorn sheep restoration. Restoration Ecology 2000, 8:25-37.

28. George JL, Martin DJ, Lukus PM, Miller MW: Epidemic pasteurellosis in a bighorn sheep population coinciding with the appearance of a domestic sheep. Journal Wildlife Diseases 2008, 44:388-403.

29. Rudolph KM, Hunter DL, Rimler RB, Cassirer EF, Foreyt WJ, Delong WJ, Weiser GC, Ward ACS: Microorganisms associated with a pneumonic epizootic in Rocky Mountain bighorn sheep (Ovis canadensis canadensis). Journal Zoo and Wildlife Medicine 2007, 38:548-558.

doi:10.1186/1472-6785-11-5

Cite this article as: Boyce et al:: Wildlife translocation: the conservation implications of pathogen exposure and genetic heterozygosity. BMC Ecology 2011 11:5.

\section{Submit your next manuscript to BioMed Central and take full advantage of:}

- Convenient online submission

- Thorough peer review

- No space constraints or color figure charges

- Immediate publication on acceptance

- Inclusion in PubMed, CAS, Scopus and Google Scholar

- Research which is freely available for redistribution

Submit your manuscript at www.biomedcentral.com/submit
Ciomed Central 